\title{
Negative Blood Oxygen Level Dependence in the Rat: A Model for Investigating the Role of Suppression in Neurovascular Coupling
}

\author{
Luke Boorman, Aneurin J. Kennerley, David Johnston, Myles Jones, Ying Zheng, Peter Redgrave, and Jason Berwick \\ Department of Psychology, University of Sheffield, Sheffield S10 2TN, United Kingdom
}

\begin{abstract}
Modern neuroimaging techniques rely on neurovascular coupling to show regions of increased brain activation. However, little is known of the neurovascular coupling relationships that exist for inhibitory signals. To address this issue directly we developed a preparation to investigate the signal sources of one of these proposed inhibitory neurovascular signals, the negative blood oxygen level-dependent (BOLD) response (NBR), in rat somatosensory cortex. We found a reliable NBR measured in rat somatosensory cortex in response to unilateral electrical whisker stimulation, which was located in deeper cortical layers relative to the positive BOLD response. Separate optical measurements (two-dimensional optical imaging spectroscopy and laser Doppler flowmetry) revealed that the NBR was a result of decreased blood volume and flow and increased levels of deoxyhemoglobin. Neural activity in the NBR region, measured by multichannel electrodes, varied considerably as a function of cortical depth. There was a decrease in neuronal activity in deep cortical laminae. After cessation of whisker stimulation there was a large increase in neural activity above baseline. Both the decrease in neuronal activity and increase above baseline after stimulation cessation correlated well with the simultaneous measurement of blood flow suggesting that the NBR is related to decreases in neural activity in deep cortical layers. Interestingly, the magnitude of the neural decrease was largest in regions showing stimulus-evoked positive BOLD responses. Since a similar type of neural suppression in surround regions was associated with a negative BOLD signal, the increased levels of suppression in positive BOLD regions could importantly moderate the size of the observed BOLD response.
\end{abstract}

\section{Introduction}

To deliver insights into the neural processes underlying affective, cognitive and sensorimotor behavior, modern functional magnetic resonance imaging (fMRI) relies on the quantitative relationship that links local neural and hemodynamic activity. This relationship has been termed neurovascular coupling. In fMRI the most commonly reported association is between the positive blood oxygen level-dependent (BOLD) response and evoked neural activity. In cases where the brain responds to a phasic event, the positive BOLD response (PBR) is caused predominantly by a local influx of fresh oxygenated blood which flushes away the paramagnetic deoxygenated hemoglobin. It is now generally agreed that PBR correlate most closely to population-based EPSPs, evoked by afferent signals (Logothetis and Pfeuffer, 2004; Logothetis and Wandell, 2004; Thomsen et al., 2004; Rauch et al., 2008).

An often observed but less frequently reported fMRI signal in both human (Shmuel et al., 2002; Smith et al., 2004; Northoff et al., 2007; Pasley et al., 2007; Kastrup et al., 2008) and animal

Received Dec. 8, 2009; revised Feb. 4, 2010; accepted Feb. 9, 2010.

We gratefully acknowledge the support of the MRC New Investigator Grant G0601,581. We thank the technical staff of the psychology department: Marion Simkins, Natalie Kennerley, and Michael Port.

Correspondence should be addressed to: Dr. Jason Berwick, Department of Psychology, University of Sheffield, Sheffield S10 2TN, UK. E-mail: j.berwick@sheffield.ac.uk.

DOI:10.1523/JNEUROSCI.6063-09.2010

Copyright $\odot 2010$ the authors $\quad 0270-6474 / 10 / 304285-10 \$ 15.00 / 0$ investigations (Harel et al., 2002; Shmuel et al., 2006; Alonso Bde et al., 2008) is a negative BOLD response (NBR). It is usually, but not exclusively found in regions adjacent to areas of increased BOLD activity. An important study in primate visual cortex by Shmuel et al. (2006) showed that the NBR correlated with local decreases in neuronal activity. However, it has been suggested that negative responses could be generated by vascular mechanics rather than opposing changes in neuronal activity (Harel et al., 2002). Resolution of this issue is critical if human fMRI experiments are to be interpreted appropriately. This point was emphasized recently in a plea for better descriptions of the neurovascular coupling relationships that link neural excitations/ inhibitions with bidirectional changes in BOLD activity (Editorial, 2009). The present study addresses this issue directly. To provide a quantitative analysis of neurovascular coupling, we have chosen a preparation in which neural activation can be controlled precisely. The somatosensory barrel cortex of the rodent has for many years been used as the preparation of choice for this purpose. Thus, the precise spatial topography of this region and surrounding cortical areas representing forelimbs, trunk and hind limbs are ideal for analyzing the relationship between neural and hemodynamic signals. Electrical stimulation of the whisker pad, in the urethane anesthetized rat, was used to evoke controlled patterns of neural activation in barrel cortex. Associated hemodynamic changes were measured with fMRI, optical imaging spectroscopy and laser Doppler flowmetry. In this preparation areas of negative BOLD signaling were observed 
not only contralaterally in regions adjacent to barrel cortex, but also within the ipsilateral barrel region. The NBR were confined to deeper layers of the cerebral cortex while PBR in the barrel region occurred more superficially. The regions of NBR were associated with locally reduced blood flow and volume which in turn, caused locally increased levels of deoxyhemoglobin. Finally, the hemodynamic changes responsible for local NBR were associated with locally decreased levels of multiunit spiking activity.

\section{Materials and Methods}

\section{Animal preparation and surgery}

Female Hooded Lister rats of 230-330 g were kept in a $12 \mathrm{~h}$ dark/light cycle at a temperature of $20^{\circ} \mathrm{C}$, with food and water supplied ad libitum. Animals were anesthetized with urethane at $1.25 \mathrm{~g} / \mathrm{kg}$ i.p., additional doses of $0.1 \mathrm{ml}$ of urethane were administered if required. Atropine was administered at $0.4 \mathrm{mg} / \mathrm{kg}$ s.c. to lessen mucous secretions during surgery. Temperature was maintained at $37^{\circ} \mathrm{C}$ using a homoeothermic blanket (Harvard Apparatus) through rectal temperature monitoring during surgery and experimental procedures. The animals were tracheotomized, allowing artificial ventilation and recording of end-tidal $\mathrm{CO}_{2}$. Blood gas measurements and end-tidal $\mathrm{CO}_{2}$ measurements were taken to allow cor-

rect adjustment of ventilator parameters to keep the animal within normal physiological limits. Both the left and right femoral arteries and veins were cannulated to allow the measurement of mean arterial blood pressure (MABP) and drug infusion. Phenylephrine was infused at $0.13-0.26 \mathrm{mg} / \mathrm{h}$ to maintain MABP between 100 and 110 $\mathrm{mmHg}$ (Golanov et al., 1994; Nakai and Maeda, 1999). All procedures were performed in accordance with the 1986 Animal (Scientific Procedures) Act, under approval from the UK Home Office.

For all experiments nonmagnetic platinum stimulation electrodes insulated to within $2 \mathrm{~mm}$ of the tip were inserted in a posterior direction between rows $A / B$ and $C / D$ of the left whisker pad of the rat thereby ensuring the whole whisker pad was stimulated. The electrical stimulation parameters used throughout were $1.2 \mathrm{~mA}$, frequency $5 \mathrm{~Hz}$ for $16 \mathrm{~s}$. A $5 \mathrm{~Hz}$ stimulation frequency is known to result in the greatest magnitude of hemodynamic responses in the somatosensory cortex of the anesthetized rodent preparation (Martin et al., 2006), without producing a change in $\mathrm{MABP}, \mathrm{pCO}_{2}$ or heart rate.

\section{Experiment 1: BOLD fMRI}

Animals ( $n=8$ for coronal, $n=6$ for tangential imaging) were placed in a magnet compatible capsule and held secure with ear and nose bars. The capsule was fixed inside the magnet using a rail system (Bruker). A surface coil (20 mm ${ }^{1} \mathrm{H}$ Bruker 1P T7399) was secured in a custom frame, contacting the skin directly above the cortical surface. An isolated MRI compatible electronic homoeothermic heating blanket (Harvard Apparatus) with rectal probe maintained the animal's body temperature at $37^{\circ} \mathrm{C}$. The animal was artificially ventilated (Zoovent) and a pressuresensitive pad was placed under the rat to monitor breathing (SA Systems, Small Animal Monitoring). Mean arterial blood pressure was monitored with a transducer connected to a receiver box (CWE Systems). A ${ }^{1} \mathrm{H}$ quadrature volume resonator (Bruker 1P-T9561, $300 \mathrm{MHz}, 1 \mathrm{~kW}$ max, outer diameter $200 \mathrm{~mm}$ /inner diameter $200 \mathrm{~mm}$ ) was placed at the isocenter of a 7 tesla magnet (Bruker BioSpecAvance, $310 \mathrm{~mm}$ bore, MRI system B/C 70/30). (error bars, SEM; scale bar, $5 \mathrm{~mm}$ ).

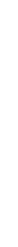

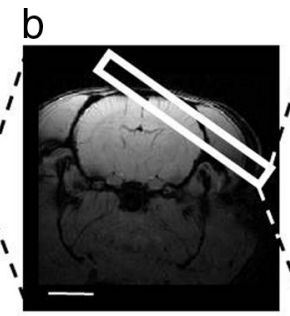
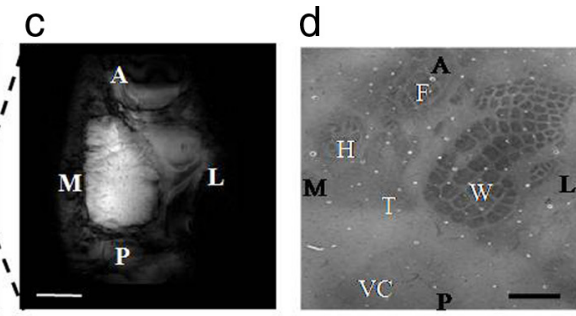

f
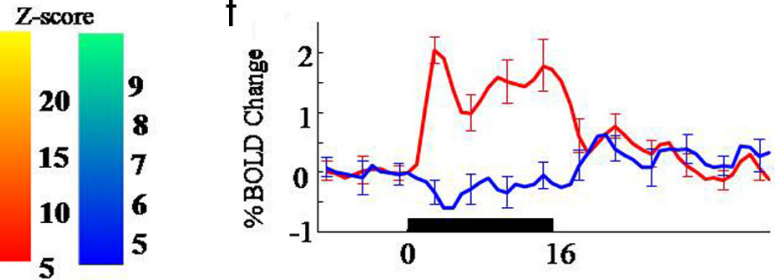
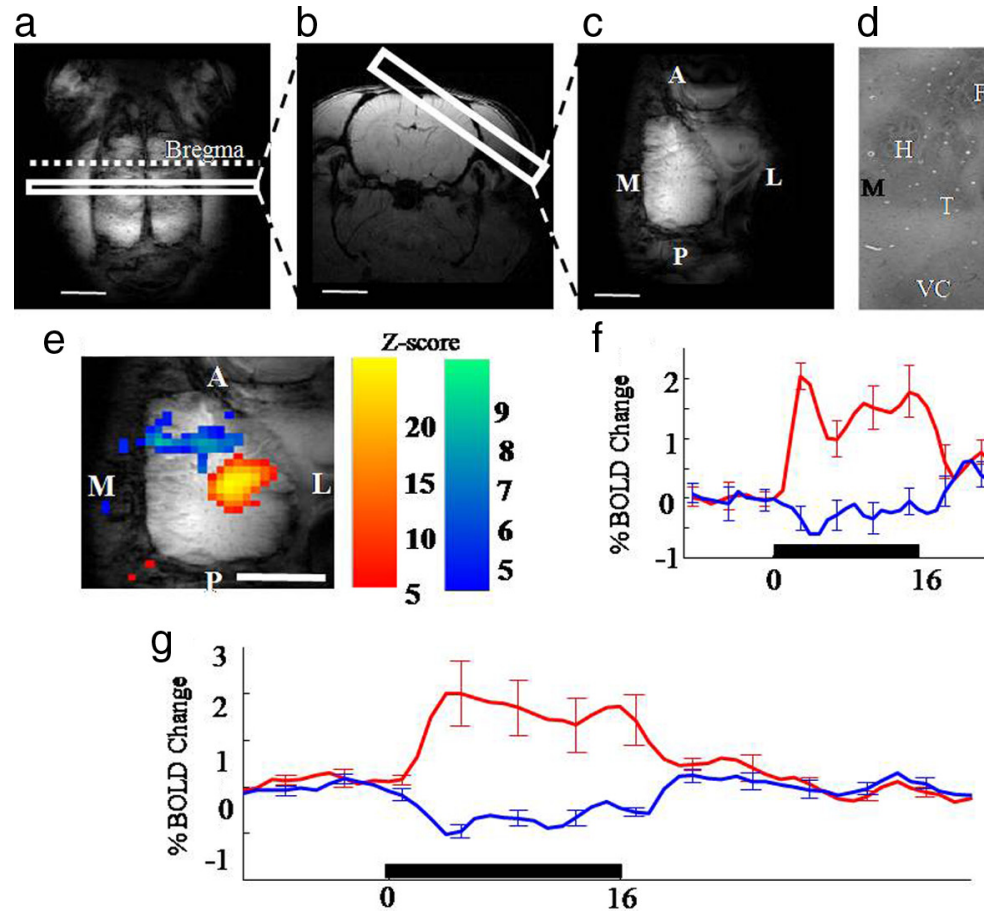

Figure 1. $\quad \boldsymbol{a}-\boldsymbol{d}$, Structural MRl images to define image orientations of transverse $(\boldsymbol{a})$, coronal $(\boldsymbol{b})$, or tengential image planes $(\boldsymbol{c})$ , $5 \mathrm{~mm}$ ), and cytochrome oxidase-stained layer IV (d) rat somatosensory cortex. Orientation: $A$, Anterior; L, lateral; $M_{,}$ image plane.f, Trial-averaged time series responses taken from the PBR and NBR regions. $\boldsymbol{g}$, Average PBR and NBR across 6 animals

The volume and surface resonators were tuned and matched to 300 $\mathrm{MHz}$ using the Multlink ${ }^{1} \mathrm{H}$ preamplifier with a built-in tune/match display. Both coils were connected to an active decoupling coil control unit operated from the console using a DC/pulse-in to ensure accurate transmit/receive times. High spatial resolution gradient echo scans were performed $\left(256^{\star} 256\right.$ pixels, FOV $=30 \mathrm{~mm}$, slice thickness $=2 \mathrm{~mm}, \mathrm{TR} / \mathrm{TE}=1000 / 15 \mathrm{~ms}$, flip angle $=90^{\circ}, 2$ averages $)$. A transverse slice was used to identify the position of bregma from which a coronal scan $2 \mathrm{~mm}$ posterior was taken to image the whisker barrel cortex. Using this coronal section an oblique slice was identified which we have termed 'tangential' because it produces an MR image of the right cortical surface in the same plane as the twodimensional optical imaging spectroscopy (2D-OIS) experiments (Fig. 1c). This plane allows the somatotopic representation of the whisker barrel field to be observed.

During the experiment functional data were acquired from the tangential and coronal slices using a single shot MBEST gradient echoechoplanar imaging (GE-EPI) sequence (raw data matrix $=64^{\star} 64$, data sampling interval $=5 \mu \mathrm{s}, \mathrm{FOV}=30 \mathrm{~mm}$, slice thickness $=2 \mathrm{~mm}$, $\mathrm{TR} / \mathrm{TE}=1000 / 12 \mathrm{~ms}$, flip angle $90^{\circ}, 10$ dummy scans). Read-out direction was left-right for both slice orientations. The left whisker pad was electrically stimulated at an intensity of $1.2 \mathrm{~mA}$, frequency $5 \mathrm{~Hz}$ for $16 \mathrm{~s}$. The majority of the BOLD experiments ( $4: 6$ animals) consisted of collecting baseline data for $60 \mathrm{~s}$ followed by $7 \times 16 \mathrm{~s}$ stimulations each separated by $96 \mathrm{~s}$. For the remaining two animals, the $60 \mathrm{~s}$ baseline was followed by $30 \times 16 \mathrm{~s}$ stimulations each $70 \mathrm{~s}$ apart. The BOLD signal was calculated as fractional change normalized by the baseline mean, recorded during the initial $1 \mathrm{~min}$ control period. All stimulus control was performed using a CED1401 which was stimulus locked to MR echo acquisition.

Data analysis was performed using MATLAB (The Mathworks). The functional GE-EPI images were structurally registered to GE anatomical images. Statistical analysis was performed using the general liner model (GLM) SPM approach (Friston et al., 1991). The time series of each pixel 
was compared with a design matrix of a DC offset, ramp and a gamma function representing the hemodynamic response function. This allowed voxel-by-voxel calculation of activation $\mathrm{Z}$ scores. Thresholding criteria of five adjacent active voxels, each with a $z$-score $>4.0$ were used to identify regions of interest, which were overlaid onto high resolution structural scans. The time series were then averaged across trials and subjects.

\section{Experiment 2: 2D-OIS}

Animals $(n=11)$ were placed in a stereotaxic frame (Kopf Instruments). The skull overlying somatosensory cortex was thinned to translucency with a dental drill; the skull surface cooled with saline during drilling. A circular plastic 'well' (20 mm diameter) was positioned over the thinned area of the skull and attached with dental cement. To reduce specularities reflecting from the skull surface the well was filled with saline.

A Dalsa $1 \mathrm{M} 30 \mathrm{P}$ camera operating in $4 \times 4$ binning mode recorded the images with each pixel representing $75 \times 75 \mu \mathrm{m}$ of the object. The camera's quantum efficiency was $28 \%$ at $500 \mathrm{~nm}$. To generate spatial maps of cortical hemodynamic responses, 2D-OIS was performed using a Lambda DG-4 high-speed filter changer (Sutter Instrument Company). The 4 wavelengths were specifically chosen as 2 pairs $(495 \pm 31 \mathrm{~nm}$ FWHM and $559 \pm 16 \mathrm{~nm}$ FWHM; $575 \pm 14 \mathrm{~nm}$ FWHM, and $587 \pm 9 \mathrm{~nm}$ FWHM) such that each pair had a similar total absorption coefficient (therefore sampled the same tissue volume) but had absorption coefficients for oxyhemoglobin $\left(\mathrm{HbO}_{2}\right)$ and deoxyhemoglobin ( $\mathrm{Hbr}$ ) that were as different as possible to maximize signal-to-noise ratios. The frame rate of the camera was $32 \mathrm{~Hz}$, which was synchronized to the filter switching. This gave an effective frame rate of $8 \mathrm{~Hz}$ for each wavelength and corresponding frequency estimates of hemodynamic changes. Spectral analysis was based upon the path length scaling algorithm (PLSA) described previously (Berwick et al., 2005, 2008). Briefly, the algorithm used modified BeerLambert Law with a path length correction factor. We estimated the concentration of hemoglobin in tissue at a concentration $104 \mu \mathrm{M}$ based on previous measurements (Kennerley et al., 2005) and saturation was calculated on a pixel by pixel basis (Berwick et al., 2008). The spectral analysis produced 2D images over time, of $\mathrm{HbO}_{2}, \mathrm{Hbr}$, and total blood volume ( $\mathrm{Hbt}$ ). The effects of electrical stimulation of the whisker pad on intrinsic signal hemodynamics were measured with 2D-OIS using a similar experimental design as that used for the fMRI experiment described above. The electrical stimulus parameters were the same $(1.2 \mathrm{~mA}$ for $16 \mathrm{~s}$ at $5 \mathrm{~Hz})$ with each experiment consisting of $30 \times 16 \mathrm{~s}$ trials separated by an interval of $70 \mathrm{~s}$. Individual trials were averaged to create a 'mean' trial which was subjected to spectral analysis described above.

\section{Experiment 3: electrophysiology and laser Doppler flowmetry}

Multichannel electrodes with LDF probes attached were inserted into the center of the regions showing the greatest increases and decreases in $\mathrm{Hbt}$, determined by prior 2D-OIS (Fig. $2 e, f$ ). In 4 cases probes were located in surround and whisker regions, while in a further 3 animals they were in the surround region alone. Extra data from two animals for whisker (making a total of $n=6$ ) and one in surround region (making $n=8$ ) were included in the absence of LDF to generate the field potential data (see Fig. 6). Electrodes were inserted through small holes drilled in the previously thinned skull. The 16 channel electrode probe (100 $\mu \mathrm{m}$ spacing, area of each site $177 \mu \mathrm{m}^{2}, 1.5-2.7 \mathrm{M} \Omega$ impedance, $33 \mu \mathrm{m}$ width at tip, Neuronexus Technologies), was inserted normal to the cortical surface, to a depth of $1500 \mu \mathrm{m}$. The probe was coupled to a preamp and data acquisition device (Medusa Bioamp, Tucker-Davis Technologies) operating a custom written MATLAB script (The Mathworks). The 2D-OIS experiment described above was then repeated with simultaneous electrophysiological recording.

The 16-channel neural data were subjected to (1) current source density analysis, to provide a measure of the incoming synaptic activity, and (2) spectral power analysis, to measure changes in overall power of the neural response in local field potential and multiunit frequency bands.
Current source density analysis. Recordings were averaged over trials, with stimulus onset "jittered" within a 20 ms window to reduce effects of $50 \mathrm{~Hz}$ mains noise. Evoked field potential recordings were sampled at 6 $\mathrm{kHz}$ with 16-bit resolution. The CSD analysis has been described in detail previously (Martindale et al., 2003), in which the field potentials are used to obtain spatiotemporal estimates of the current sources and sinks within the cortical layers (Nicholson and Freeman, 1975; Mitzdorf, 1985).

Frequency analysis. To provide further information concerning electrophysiological responses evoked in areas of increased/decreased hemodynamic activity, an analysis of the power in different frequency bands was performed (Shmuel et al., 2006). Data were accumulated in $1 \mathrm{~ms}$ bins and Fourier transformed. Power in the ranges $30-130 \mathrm{~Hz}$ (local field potentials) and $500-3000 \mathrm{~Hz}$ (multiunit) for each bin was calculated and averaged across trials and animals.

\section{Cytochrome oxidase histochemistry}

Following each experiment rats were perfused transcardially with saline, followed by $4 \%$ paraformaldehyde and finally photographic emulsion (Fotospeed Ltd). The latter enables surface cortical vessels to be observed in histological tissue. Brains were removed and the right cortex was separated and compressed to a thickness of $\sim 2 \mathrm{~mm}$. Tangential slices of frozen cortical tissue were cut in a cryostat. An initial slice of $200 \mu \mathrm{m}$ was taken to include surface vasculature after which the sections were $50 \mu \mathrm{m}$. Sections were placed into an incubation medium in a dark room at $37^{\circ} \mathrm{C}$ and stained for cytochrome oxidase using a modified version of the procedure described by Wong-Riley and Welt (1980). Photographic emulsion in the blood vessels was developed after staining. Photomicrographs of histological sections were taken on a Nikon Eclipse microscope $(10 \times$ magnification) and the images linearly warped by locating corresponding features.

\section{Results \\ BOLD responses in rat somatosensory cortex}

fMRI was used to record hemodynamic changes in the rat somatosensory cortex in response to a train of electrical stimulation $(16 \mathrm{~s}, 5 \mathrm{~Hz})$ applied unilaterally to the left whisker pad of urethane anesthetized rats. The regional specificity PBR and NBR was assessed: (1) in a contralateral tangential plane to determine the location of BOLD responses with respect to the topographical organization of somatosensory cortex (Fig. 1c); and (2) in a coronal plane to locate the BOLD responses dorsoventrally within the layered structure of cerebral cortex and to detect responses ipsilateral to the sensory stimulation (Fig. 1b).

In the tangential plane (Fig. 1e) electrical stimulation of the whisker pad evoked a PBR confined to the barrel field, and an NBR anterior and medial to the whisker barrel field, located in forelimb, hindlimb and trunk regions of somatosensory cortex (Fig. 1d). Averaged time series from an individual subject (Fig. 1f) showed the PBR response initially peaked 3-4 s after stimulus onset falling back to a plateau that was maintained until the end of the stimulation period, at which point a further $5 \mathrm{~s}$ was required for the response to return to baseline. Although the magnitude of the NBR was $\sim 75 \%$ smaller, the temporal characteristics of the negative response were similar but opposite to the PBR (Fig. $1 f$ ). The generality of the PBR and NBR for single subject was confirmed in group data $(n=6$, Fig. $1 g)$.

In experiments using a coronal image plane, stimulus-evoked PBR and NBRs were observed in the cortex (Fig. 2a). Again the PBR was confined to the contralateral whisker barrel field while NBRs were observed in adjacent tissue and in ipsilateral barrel cortex. The NBR was located ventrolaterally in secondary somatosensory cortex (Fig. 2a), which would fall below the previously used tangential plane and therefore failed to appear in Figure 1a. Analysis of time series data confirmed the PBR and 
NBR patterns observed in the tangential plane in both individual (Fig. 2b) and group data (Fig. 2c). The main discovery from coronal plane data (Fig. $2 a, d, e$ ) was that NBRs were located in the deep layers of cerebral cortex (predominantly in layers V-VI) while the PBR was located superficially (mainly in layers I-IV). Thus, plotting the $z$-scores along lines dropping down through the center of the contralateral PBR and NBR (Fig. 2d) for each subject, showed the average depth for the mean maximum positive $z$-score was $0.5 \mathrm{~mm}$ (corresponding to layer IV), while the average depth of the mean maximum negative $z$-score was $1.3 \mathrm{~mm}$ (corresponding to layers V/VI). These mean depth values for the maximum PBR and NBR were reliably different $(T=-8.07$; $\mathrm{df}=7 ; p<0.0001)$.

These results confirm that rat somatosensory cortex is a good experimental model to investigate bidirectional BOLD responses. Moreover, they show that evoked neural activity can induce spatially discrete patterns of PBR and NBR, but at different locations within the layered cortical micro-circuitry.

\section{Optical measurements of \\ hemodynamic responses}

It is however, important to recognize that the BOLD signal measured by fMRI is complex, where changes in the ratio of $\mathrm{HbO}_{2}$ and $\mathrm{Hbr}$ are confounded with local changes in blood volume. Therefore, we used two-dimensional optical imaging spectroscopy (2D-OIS) in the same experimental paradigm to examine further the extent to which PBR and NBR may be influenced differentially by blood volume and saturation. The spatial resolution of this technique can provide simultaneous measures of changes in $\mathrm{HbO}_{2}, \mathrm{Hbr}$ and $\mathrm{Hbt}$ which can be localized to individual blood vessels.

In a second group of subjects $(n=11)$, we positioned a CCD camera over a thinned cranial window, which provided a clear view of the cortical surface and associated vasculature (Fig. $3 a, b$ ). Intrinsic signals were recorded at four wavelengths $(495,559$, 575 , and $587 \mathrm{~nm}$ ) and the resulting optical data subjected to spectroscopic analysis. Spatial images of $\mathrm{HbO}_{2}, \mathrm{Hbr}$, and $\mathrm{Hbt}$ over time were generated Hemodynamic responses to the same electrical stimulation of the contralateral whisker pad plotted over time are presented in a series of montage images from a single subject (Fig. 3a). To interpret these images it is important to have a good understanding of the geometry of the surface vasculature that supplies blood to, and drains blood from, the somatosensory cortex (Fig. $3 a, b$ ). Therefore, the location of the barrel field with respect to the vasculature in this case is also presented (Fig. $3 d$ ). The red lines in Figure $3 c$ denote branches of the middle cerebral artery (MCA) while blue lines highlight the draining veins. Comparing Figure 3, c and $d$, it will be noted that only branch 1 of the MCA supplies whisker cortical layers.
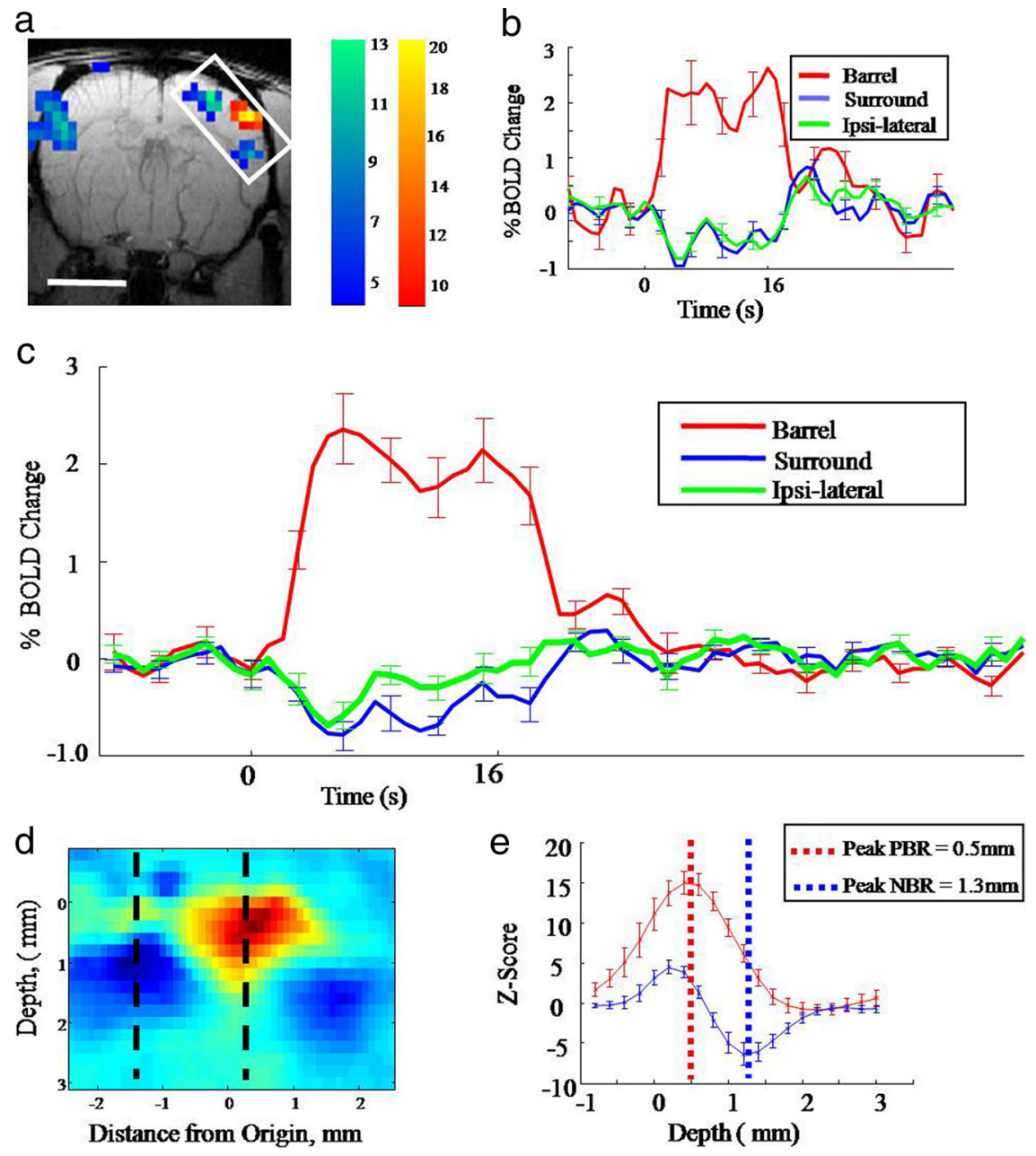

Figure 2. Representative $\mathrm{AMRI} B O L D$ results in the coronal image plane. $\boldsymbol{a}$, Activation map for a representative animal showing PBR and NBR in response to a $16 \mathrm{~s}$ whisker stimulation (scale bar, $5 \mathrm{~mm}$ ). $\boldsymbol{b}$, Trial-averaged time series responses taken from the PBR in $\boldsymbol{a}$; this shows that the NBR appears to originate from a deeper cortical location than the PBR. $\boldsymbol{e}, Z$-scores as a function of depth for both the NBR and PBR across all animals. The PBR peaks at a depth of $0.5 \mathrm{~mm}$, the NBR peaks at a depth of $1.3 \mathrm{~mm}$ in the deeper

barrel cortex. The two anterior branches supply other regions of cortex including representations of the forepaw (branch 2) and motor cortex (branch 3 ).

Electrical stimulation of the contralateral whisker pad induced hemodynamic changes (Fig. $3 a$ ) with the same spatial and temporal characteristics as those of the BOLD responses illustrated in Figure 1. Within 4 s of stimulus onset a clear increase in Hbt was observed in MCA branch 1 with a smaller decrease in $\mathrm{Hbt}$ in MCA branch 2. These changes were accompanied by increases (warm colors) in $\mathrm{Hbt}$ and $\mathrm{HbO}_{2}$ and decreases (cold colors) in $\mathrm{Hbr}$ that were confined to the barrel field surrounding MCA branch 1. Similar but opposite regional changes in these hemodynamic variables were recorded rostromedially in somatosensory cortex supplied by MCA branch 2 . Both the positive and negative hemodynamic responses were largely maintained throughout the $16 \mathrm{~s}$ stimulus train, after which they returned to near baseline values in the following $4 \mathrm{~s}$.

To demonstrate the generality of this pattern, responding parenchymal regions directly adjacent to the arteries showing the largest increases and decreases in Hbt were selected for analysis in 11 subjects. The mean temporal profile of evoked responses in the regions of increased Hbt (whisker barrel cortex-Fig. 4a) and 
a Stimulation

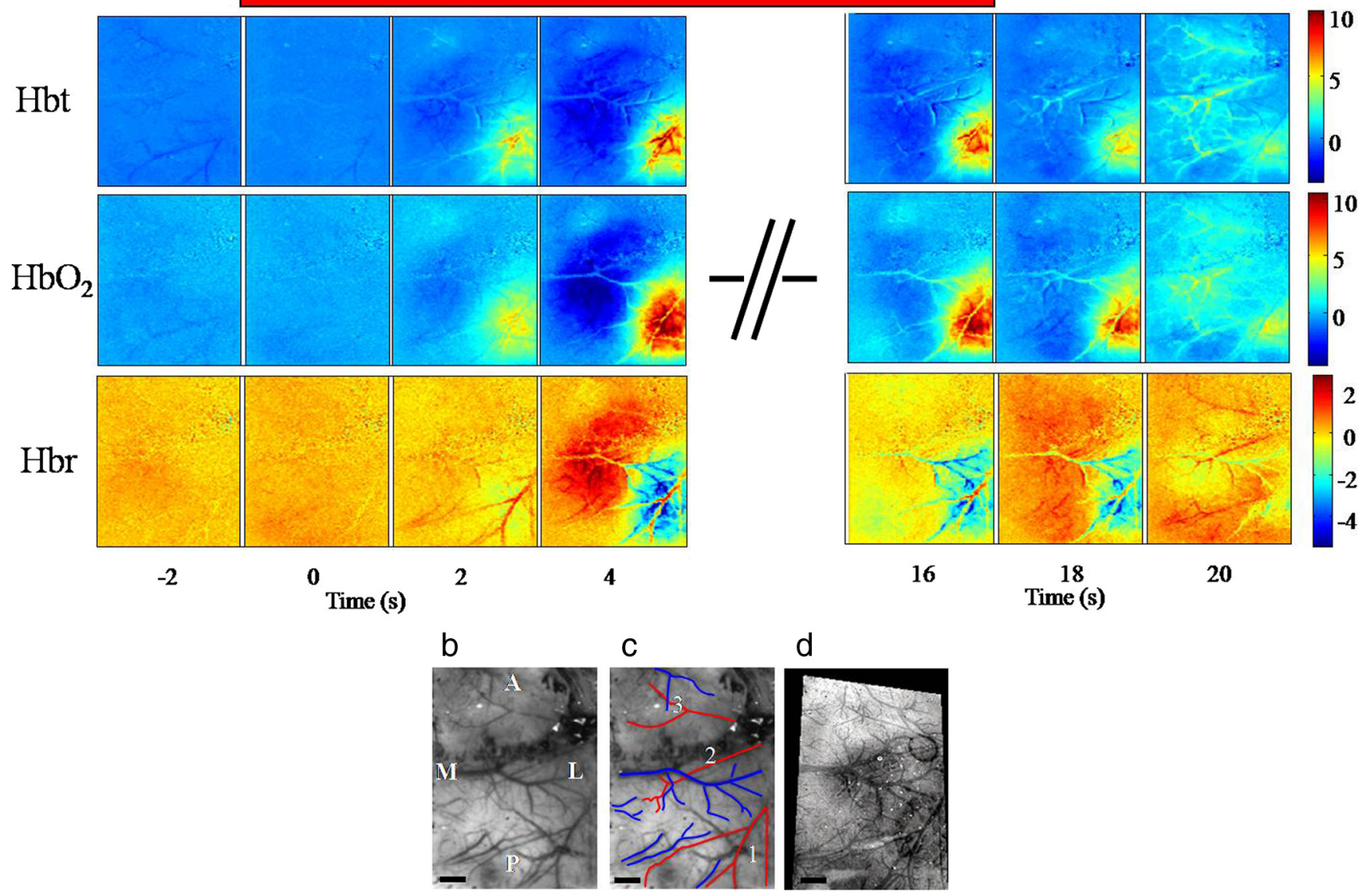

Figure 3. The hemodynamic response for (16s) electrical whisker stimulation ( $1.2 \mathrm{~mA}$ at $5 \mathrm{~Hz}) . \boldsymbol{a}$, Image montage for $\mathrm{Hbt}, \mathrm{HbO}_{2}$, and $\mathrm{Hbr}$. Each image represents a snapshot in time before, during, and after the $16 \mathrm{sstimulus.} \mathrm{The} \mathrm{scale} \mathrm{bar} \mathrm{represents} \mathrm{the} \mathrm{micromolar} \mathrm{change} \mathrm{from} \mathrm{baseline.} \mathrm{Stimulation} \mathrm{occurs} \mathrm{at} \mathrm{time} 0 . \boldsymbol{b}, \boldsymbol{c}$, In vivo images of the surface vasculature show the branches of the middle cerebral artery (red) and draining veins (blue). Branch 1 of the artery supplies the whisker barrel cortex, branch 2 the forepaw region, and branch 3 the motor cortex in front of bregma. $\boldsymbol{d}$, The relationship of the barrel cortex to the surface vasculature is shown in a combined postmortem image. This postmortem image was fitted to the in vivo image so that the barrels could then be superimposed over the activation maps as a reference. M, Medial; L, lateral; A, anterior; P, posterior. Scale bar, $1000 \mu \mathrm{m}$.

decreased Hbt (surrounding areas-Fig. $4 b$ ) were reminiscent of the respective spatiotemporal changes in PBR and NBR elicited by the same sensory stimulus (Figs. 1c, 2c). Together these results confirm that the predominant cause of PBR is a regional increase in oxygenated blood and a washing away of $\mathrm{Hbr}$, while the NBR is produced mainly by a regional decrease in blood volume and associated elevation of $\mathrm{Hbr}$.

However, these results leave two important issues outstanding with respect to the antecedent causes of regional reductions in blood volume. First, reductions in blood volume are normally considered a consequence of local reductions in blood flow; and second, to what extent may such changes be associated with corresponding reductions in local neuronal activity. Therefore, in the final part of the study we sought to address these issues by measuring simultaneously both blood flow with laser Doppler flowmetry (LDF) and local neural activity with concurrent electrophysiological recordings.

\section{Concurrent measurements of neural activity and local cerebral blood flow}

At the beginning of each experiment 2D-OIS was used to identify regions of positive and negative Hbt (Fig. 5a) to which LDF probes and recording electrodes could subsequently be directed (Fig. 5b). The LDF probes (Periflux) were attached to two 16channel recording electrodes (100 $\mu \mathrm{m}$ spacing, NeuroNexus
Technologies Inc). With the electrodes and LDF probes in place, the same $16 \mathrm{~s}$ electrical stimulation of the whisker pad was again used to evoke neural and hemodynamic responses.

\section{Laser Doppler flowmetry}

Corresponding positive and negative changes in blood flow were observed in the regions of positive $\mathrm{Hbt}(n=4)$ and negative $\mathrm{Hbt}$ $(n=7)$ respectively (Fig. $5 c)$. The sign and time course of bloodflow changes in the positive and negative regions were similar to those reported previously for BOLD (Figs. 1, 2) and Hbt recorded with 2D-OIS (Fig. 4).

\section{Multichannel electrophysiology}

The multichannel recording electrodes were inserted normal to the cortical surface, to a depth of $1600 \mu \mathrm{m}$ in regions of positive or negative Hbt responses (Fig. $5 b$ ). The signals from each of the 16 electrodes served as input to a data acquisition device (Tucker-Davis Technologies) where they were analyzed in terms of: (1) local field potentials; (2) current source density (CSD) (Nicholson and Freeman, 1975; Mitzdorf, 1985) and (3) analysis of power present in the low $(30-130 \mathrm{~Hz}-$ local field potential) and high frequency $(500-3000 \mathrm{~Hz}-$ multiunit) components (Shmuel et al., 2006).

Local field potentials. A composite image of the field potentials recorded across the cortical layers was generated by averaging 

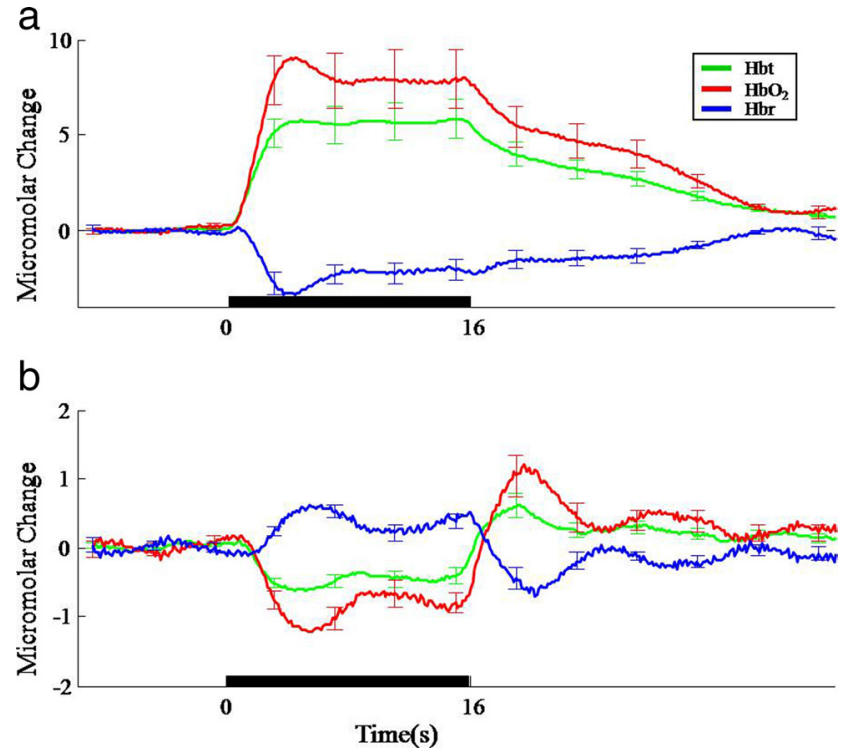

Figure 4. Average time series responses for $\mathrm{Hbt}, \mathrm{HbO}_{2}$, and $\mathrm{Hbr}$ following $16 \mathrm{~s}$ electrical whisker stimulation taken from selected regions across animals. $\boldsymbol{a}$, Parenchyma region in whisker barrel cortex. $\boldsymbol{b}$, Parenchyma from surround negHbt region (error bars, SEM).

a
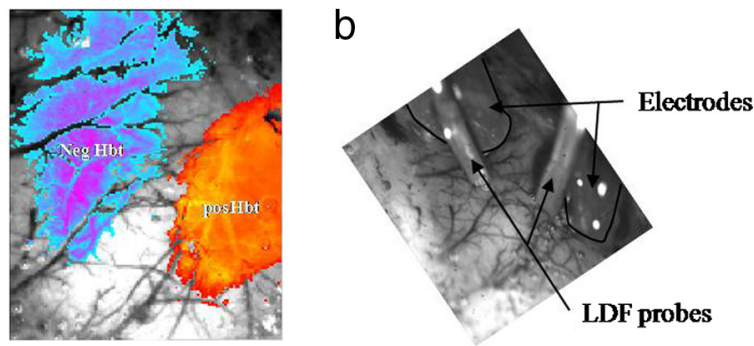

C

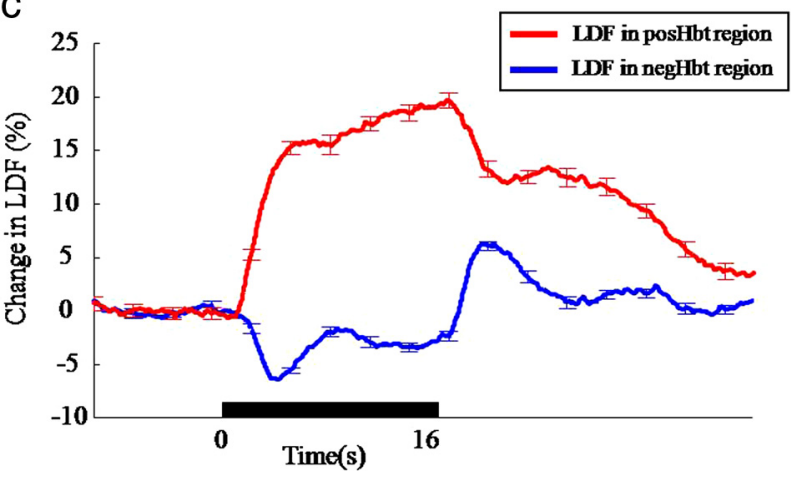

Figure 5. Placement of and response from laser Doppler flowmetry probes to $16 \mathrm{~s}$ whisker stimulation. $\boldsymbol{a}$, Activation map showing regions of increased and decreased blood volume which provided targets for the placement of 16-channel electrode and LDF probe. $\boldsymbol{b}$, Image showing electrodes inserted in, and LDF probes over the regions of increased and decreased $\mathrm{Hbt}$. c, Cerebral blood flow responses from the LDF probe placed over the pos $\mathrm{Hbt}$ and negHbt regions (error bars, SEM).

across individual pulses of the trials for each animal $(16 \mathrm{~s} \times 5$ $\mathrm{Hz}=80$ pulses) then across animals (Fig. $6 a$ ). For the contralateral barrel region (+blood flow) the image revealed a superficial P1 followed an N1 component that extended across all layers. This pattern is characteristic of the local field potential evoked in barrel cortex by whisker pad stimulation (Di et al., 1990; Barth et al., 1993). At similar levels of resolution, no comparable response was observed in adjacent regions of reduced blood flow (data not shown). However, when the images were rescaled to emphasize smaller magnitude response components (at the expense of clipping the larger ones), longer latency potentials were recorded from regions of both positive (Fig. $6 b$ ) and negative (Fig. $6 c$ ) changes in Hbt. These components evolved in the $200 \mathrm{~ms}$ following each pulse. To demonstrate the statistical reliability of the longer latency signals, for each subject, field potentials recorded from the bottom 8 channels of electrodes located in the whisker barrel and surround regions were separately averaged. We then compared the value of the mean field potential at $120 \mathrm{~ms}$ following stimulus onset with the average value for the $10 \mathrm{~ms}$ immediately preceding the electrical stimulus. In both cases the longer latency positive signal was reliably different from the averaged prestimulus values (paired $t$ test: whisker region $t=-2.758, \mathrm{df}=$ $5, p=0.04$; surround region $t=-6.105, \mathrm{df}=7, p<0.001$ ).

Current source density analysis. The current sources and sinks associated with the stimulus-evoked field potentials illustrated in Figure $6, a-c$, were identified by subsequent CSD analyses. In accordance with previous observations (Barth et al., 1993; Berwick et al., 2004, 2008; Hewson-Stoate et al., 2005; Higley and Contreras, 2007), electrical stimulation of the whisker pad induced a large amplitude current sink $\sim 500 \mu \mathrm{m}$ below the cortical surface in layer IV, with an overlying source in layer I-III (Fig. 6d). The large sink in the middle cortical layers peaking at $\sim 10 \mathrm{~ms}$ is commonly attributed to the afferent, sensory-evoked EPSP activity in layer IV. A current sink present for a further $10 \mathrm{~ms}$ was then evident in supragranular layers, the source for which was localized below in layer IV. At approximately the same time a second sink-source dipole was identified in the deeper cortical layers, most likely due to direct postsynaptic activation of layer VI (Higley and Contreras, 2007). Again, at the same level of resolution, no current sources and sinks were evident in CSD analyses of data from regions of negative blood flow. So, to investigate whether any smaller magnitude, longer latency currents were evident, the CSD data were also rescaled (Fig. $6 e, f$ ). In regions of positive blood flow longer duration $(\sim 100 \mathrm{~ms})$ sink/source dipoles were noted in layers IV, while later still, sinks were observed in layers I and V surrounded by sources. Rescaled CSD from negative blood-flow regions also revealed two short-latency $(<40 \mathrm{~ms})$ sink source pairs in layer IV and layer VI. These were followed by a broad sink in layer I with a source across all deep channels. These longer latency response profiles appear similar to long latency single whisker responses reported by Di et al. (1990). Taken together the field potential and CSD results indicated that, following the initial short-latency response evoked by each pulse, smaller magnitude but longer latency electrophysiological responses evolve in regions of both positive and negative blood flow.

Frequency-power analysis. Multichannel data from both electrodes was analyzed in $1 \mathrm{~s}$ temporal 'bins' and Fourier transformed $(30-3000 \mathrm{~Hz})$. The data for each bin were grouped into two frequency ranges; (1) the local field potential range (30-130 $\mathrm{Hz})$; and (2) the multiunit firing frequency range $(500-3000 \mathrm{~Hz})$. In whisker cortex the power increase for both frequencies was time locked to the stimulation period $(0-16 \mathrm{~s})$ (Fig. 7a,b). Responses were however, markedly different in adjacent regions of negative blood flow, where an increase in low-frequency power was largely restricted to the superficial cortical layers. In the higher multiunit frequencies whisker pad stimulation evoked a decrease in power in the deeper cortical layers with a positive rebound after stimulus termination. The dynamics of this neural response (Fig. $7 d$, blue time series) closely resembled those of the $\mathrm{CBF}$ changes recorded at the same time in the same cortical region (Fig. 8a). 

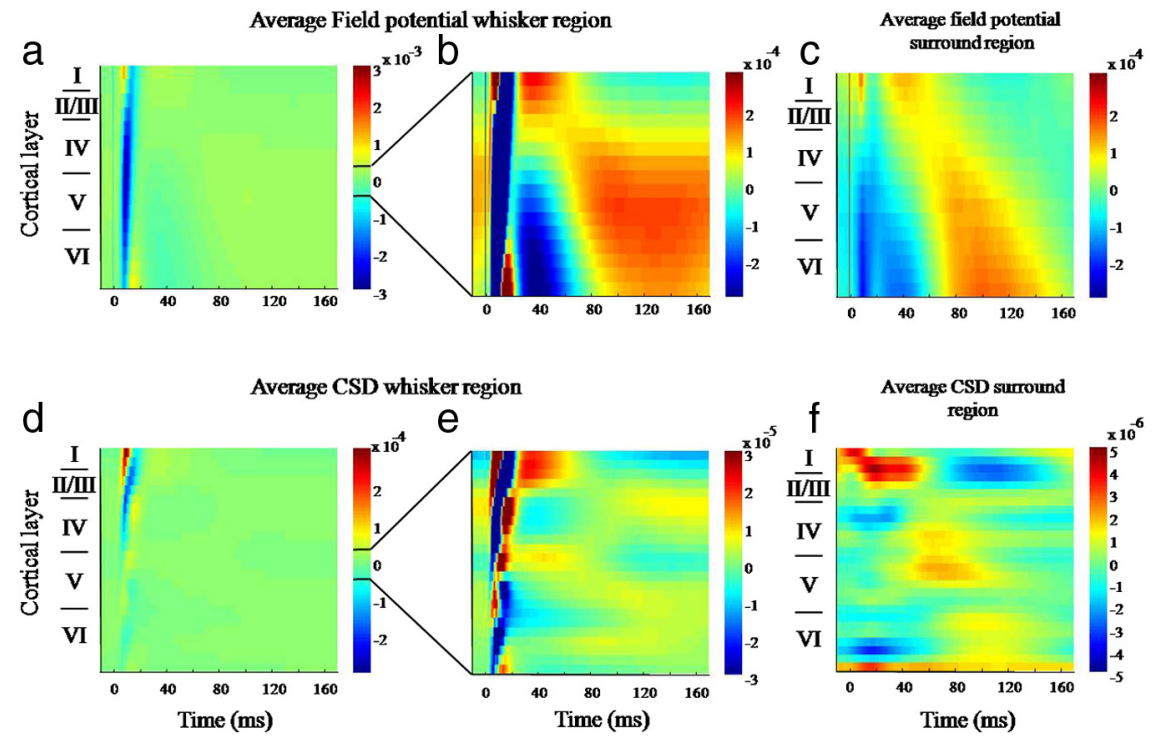

Figure 6. Field potential and current source density (CSD) responses to $16 \mathrm{~s}$ whisker stimulation in whisker barrel cortex and surrounding region. All responses represent an average of the $80(16 \times 5 \mathrm{~Hz})$ whisker deflections across the animals and trials. $\boldsymbol{a}$, Average field potential responses from the electrode inserted into the whisker region. $\boldsymbol{b}$, Same response shown in $\boldsymbol{a}$ with the threshold decreased to enable visualization of long latency responses occurring after $30 \mathrm{~ms}$. c, Field potential responses in surround region. $\boldsymbol{d}$, CSD responses from whisker barrel region. $\boldsymbol{e}$, Same response as shown in $\boldsymbol{d}$, with the threshold decreased to reveal long latency CSD responses. $f$, CSD responses from the surround region.

A cross-correlation analysis revealed reliable positive correlations between deep layer multiunit activity (high-frequency power) and CBF with a temporal lag of $1.0 \pm 0.4 \mathrm{~s}$ in surround regions for $5 / 7$ subjects $[p<0.01$ by comparing with a null distribution formed from phase-randomized surrogates (Dahlhaus et al., 2008)] (Fig. 8b). Together these findings indicate that within the region of positive blood flow, evoked electrophysiological responses indicative of increased neural activation are present. In surrounding regions of negative blood flow a significant reduction in power associated with multiunit activity was recorded in the cortical deep layers.

\section{Discussion}

The present study investigated neurovascular coupling relationships linking excitatory and inhibitory neurotransmission to positive and negative changes in BOLD signaling. Using a variety of measures to record neural and hemodynamic responses evoked by stimulation of the whisker pad, the main findings were as follows. (1) In contralateral barrel cortex NBRs were found adjacent to the evoked region of PBR. NBRs were also seen in ipsilateral cortex. (2) NBRs were located in deeper cortical layers. (3) NBRs were associated with local reductions in blood flow and volume and increased deoxyhemoglobin. (4) Following an initial short-latency electrophysiological response, longer latency field potential and CSD responses were recorded in barrel cortex and surrounding regions. (5) Hemodynamic changes underlying NBRs were associated with local reductions in multiunit activity. These findings will be discussed first in relation to human, then the animal studies that suggest associations between NBR and neural suppression. A mechanism explaining the linking of hemodynamic and electrophysiological responses will be considered.

\section{Human studies}

NBRs are observed in many human fMRI studies (Shmuel et al., 2002; Smith et al., 2004; Northoff et al., 2007; Pasley et al., 2007;
Kastrup et al., 2008). Interestingly, positive BOLD responses above baseline after stimulus cessation were also reported (Shmuel et al., 2002). In the absence electrophysiological measures, it is widely assumed that NBRs signify regions of decreased neuronal activity. However, when PBRs and NBRs are adjacent, it has been suggested that the NBR could reflect 'blood stealing' by a neighboring region of increased neural activation (Harel et al., 2002). However, it is unlikely that local hemodynamic adjustment is the sole cause of NBRs. First, NBRs and PBRs are not always in adjacent regions (Smith et al., 2004). Second, NBRs have been associated with a decrease in oxygen consumption (Shmuel et al., 2002; Stefanovic et al., 2004; Uludağ et al., 2004). Third, in visual cortex a tight coupling between $\mathrm{CBF}$ and cerebral metabolic rate of oxygen consumption during evoked responses under conditions of resting and negative BOLD suggests that NBRs provide a reliable index of suppressed neural activity (Pasley et al., 2007). Fourth, increases in somatosensory perception thresholds caused by inhibition induced by contralateral activation were associated with NBRs (Kastrup et al., 2008). Finally, a spectroscopy study (Northoff et al., 2007) reported regional correlations between levels of the inhibitory neurotransmitter GABA and NBR. Together these investigations provide indirect evidence that regions of NBR are associated with neural suppression.

\section{Animal studies}

A non-human primate study involving simultaneous fMRI and electrophysiological recording in monkeys reported direct associations between neural suppression and NBR (Shmuel et al., 2006). Trial-by-trial analysis revealed a tight coupling between NBR and decreases in local field potentials and multi-unit activity. An increase above baseline for both neural and BOLD response in the negative region after stimulation cessation was also observed. Interestingly, the NBRs in these experiments was also located in deeper cortical layers (A. Shmuel, personal communication), as were NBRs in the rat somatosensory system, following whisker deflection (Alonso Bde et al., 2008). In a related study (Devor et al., 2007), forepaw stimulation induced an area of increased hemodynamic activity in rodent somatosensory cortex surrounded by regions of decreased hemodynamic activity. The latter regions were associated with neural suppression in the form of hyperpolarization measured by voltage-sensitive dye imaging. However, the issue was complicated by a later finding of same group (Devor et al., 2008), showing that reduced levels of hemodynamic activity ipsilateral to the stimulus seemed to be associated with an increase in glucose consumption and neuronal spiking. Although we measured neuronal activation only in regions immediately surrounding the positive response, we did observe small initial increases in multiunit power immediately following stimulus onset which was subsequently supplanted by a longer lasting neuronal inhibition (Fig. 7d). An alternative mechanism for inducing NBRs was demonstrated in a rat model of epilepsy where evoked influx of CBF to discharging regions was 

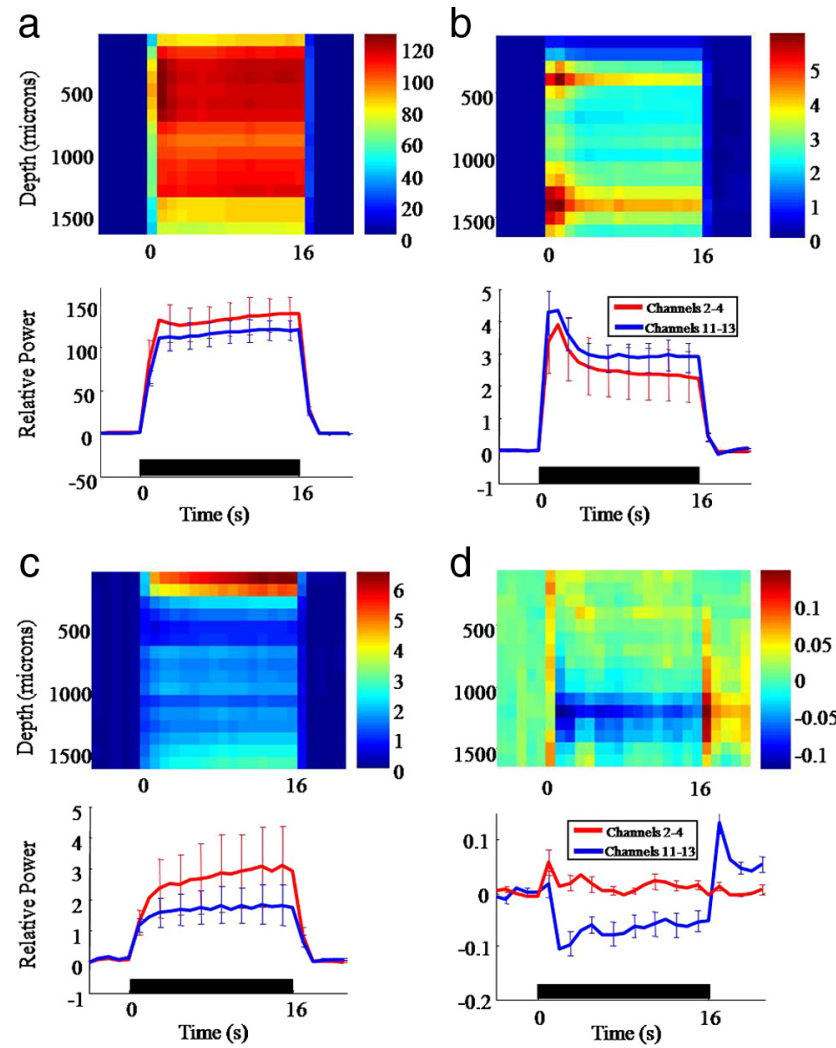

Figure 7. Frequency power analysis of 16-channel electrode recordings in the whisker and surround regions. $\boldsymbol{a}$, Top image: $30-130 \mathrm{~Hz}$ power response in $1 \mathrm{~s}$ bins for the whisker region. Bottom Image: Average time series taken through shallow (2-4) and deep (11-13) electrode channels. $\boldsymbol{b}$, Top image: $500-3000 \mathrm{~Hz}$ power response in $1 \mathrm{~s}$ bins for the whisker region. Bottom image: Average time series taken through shallow (2-4) and deep (11-13) electrode channels. c, Top image: $30-130 \mathrm{~Hz}$ power response in $1 \mathrm{~s}$ bins for the surround region. Bottom image: Average time series taken through shallow (2-4) and deep (11-13) electrode channels. $\boldsymbol{d}$, Top image: $500-3000 \mathrm{~Hz}$ power response in $1 \mathrm{~s}$ bins for the surround. Bottom image: Average time series taken through shallow (2-4) and deep (11-13) electrode channels.

unable to relieve the metabolic deficit (Schridde et al., 2008). Finally, a study modeling the vascular network claimed that vascular interaction alone could account for NBRs (Boas et al., 2008), however, their simulations significantly underestimated the magnitude of measured responses, suggesting an active mechanism may also contribute.

\section{Possible neuronal mechanisms}

An early study reporting the results of CSD analysis in rodent cortex demonstrated that stimulation of the whiskers caused an initial fast biphasic response followed by longer latency components $>100 \mathrm{~ms}$ (Di et al., 1990). It was suggested these 'slow responses' represent a 'wave' of cortical inhibition following each whisker deflection. Later, Using surface evoked potentials at multiple sites the same research group showed the long latency responses were transmitted over large areas of cortex (Barth et al., 1993). Our finding that information spreads spatiotemporally from an initial somatotopic location is consistent with these reports.

The current working hypothesis to explain our results is that stimulus-evoked excitatory and inhibitor activity are greatest in the activated region, but that the inhibitory signals spread over a greater area than the excitation (Fig. $8 c$ ). This would produce a net inhibition in surrounding regions. Importantly, the long latency field potential and CSD results for the whisker region (Fig.

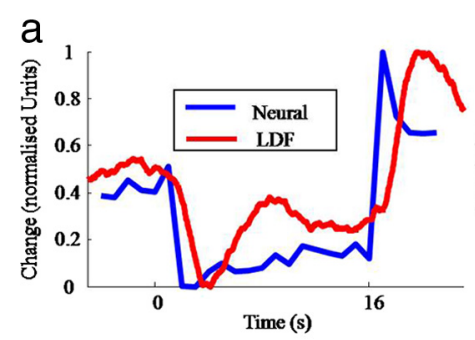

b
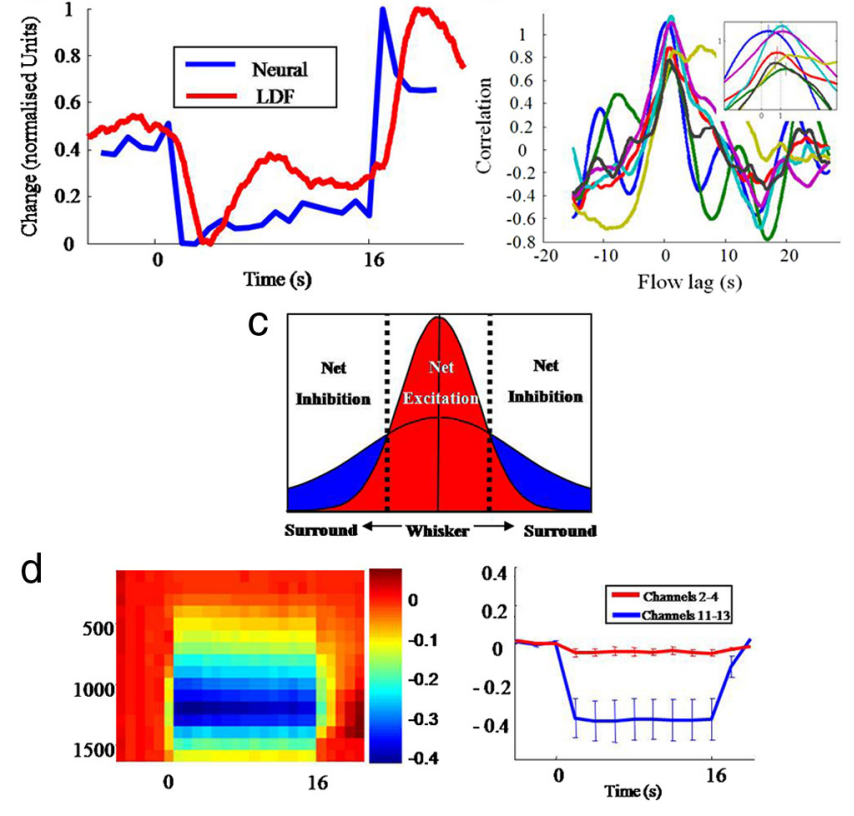

Figure 8. Simultaneous neural activation and cerebral blood flow (CBF) in surround region. $\boldsymbol{a}$, Normalized plot of CBF and $500-3000 \mathrm{~Hz}$ power time series from channels 11-13 from the surround region. $\boldsymbol{b}$, Cross-correlation analysis of individual animals at the onset of flow and neural decrease. $c$, Schematic model to explain net excitation and inhibition effects in whisker and surround regions. $\boldsymbol{d}$, Left image: $500-3000 \mathrm{~Hz}$ power analysis in the whisker region with the first $20 \mathrm{~ms}$ after each stimulus impulse removed. Right image: average time series taken through shallow (2:4) and deep (11-13) electrode channels.

$6 a, d)$ show a developing temporal suppression after each impulse of the electrical stimulus train. Because of the comparatively large early response, later inhibitory activity is not apparent in the scaled heat map of the high-frequency power analysis (Fig. 7b). To demonstrate the longer latency inhibition of multiunit activity in the activated barrel region, the first $20 \mathrm{~ms}$ of data containing the large positive response was removed and the same power analysis rerun. Importantly, the poststimulus reduction in power of the multi-unit frequencies for the whisker region was actually larger than the inhibition detected in surrounding regions (Fig. $8 d$ ). It is known that local inhibition is essential for normal cortical processing, in part to prevent uncontrolled excitation (Sun et al., 2006). Voltage sensitive dyes have been used to measure inhibition evoked by single whisker stimulation but the relative magnitudes of inhibition in surround compared with activated regions was not resolved (Derdikman et al., 2003). Our hypothesis that inhibition is largest in the central barrel region and then spreads over a wider area raises the interesting question, why is the inhibition most evident in deeper cortical layers?

\section{Deep layer inhibition}

Several mechanisms could be responsible for the decreases in neuronal activity observed in the present study. First, local inhibitory interneurons could be implicated since several anatomical studies have demonstrated long range GABAergic interactions, especially in deep cortical layers (Esclapez et al., 1987; Tomioka et al., 2005). For example, in rat visual cortex GABAergic cells at the layer V/VI border directly inhibit neurons at distant points of the topographic map (McDonald and Burkhalter, 1993). Similarly, an analysis of intracortical axonal projections of layer VI cells in $S 1$ cortex reported a dense local network of corticocortical communication in which GABAergic basket cells featured 
prominently (Zhang and Deschênes, 1997). Moreover, since GABAergic neurons project diffusely into somatosensory cortex in the opposite hemisphere (Gonchar et al., 1995), GABAergic projections may also mediate neural suppression associated with ipsilateral NBR. Alternatively, subcortical inhibitory circuitry, for example, via reentrant loops through the basal ganglia (Alexander et al., 1986), may also play a role. In this case, observed decreases in activity in the cortical microcircuits would reflect more an indirect suppression of excitatory inputs from the thalamus rather than any direct inhibitory action (Chevalier and Deniau, 1990).

In summary, electrical stimulation of the whisker pad evoked short latency neural excitation and a positive hemodynamic response of contralateral barrel cortex, which resulted in the PBR. At the same time there was a deep layer inhibitory response that spread more widely into adjacent regions and to ipsilateral cortex. In the absence of masking excitatory neural and hemodynamic responses, neural suppression in adjacent and ipsilateral regions is responsible for the observed NBRs. Finally, in a recent human study (Muthukumaraswamy et al., 2009), the tonic level of GABA in the visual cortex correlated with reduced BOLD responses. This raises the possibility that the PBR in activated regions may be subject to inhibitory modulation. Thus, a better understanding of the neurovascular coupling between neural suppression and NBR will facilitate an appreciation of how inhibition and excitation might interact in areas responding with net increases in BOLD activity.

\section{References}

Alexander GE, DeLong MR, Strick PL (1986) Parallel organization of functionally segregated circuits linking basal ganglia and cortex. Annu Rev Neurosci 9:357-381.

Alonso Bde C, Lowe AS, Dear JP, Lee KC, Williams SC, Finnerty GT (2008) Sensory inputs from whisking movements modify cortical whisker maps visualized with functional magnetic resonance imaging. Cereb Cortex 18:1314-1325.

Barth DS, Kithas J, Di S (1993) Anatomic organization of evoked potentials in rat parietotemporal cortex: somatosensory and auditory responses. J Neurophysiol 69:1837-1849.

Berwick J, Redgrave P, Jones M, Hewson-Stoate N, Martindale J, Johnston D, Mayhew JE (2004) Integration of neural responses originating from different regions of the cortical somatosensory map. Brain Res 1030:284-293.

Berwick J, Johnston D, Jones M, Martindale J, Redgrave P, McLoughlin N, Schiessl I, Mayhew JE (2005) Neurovascular coupling investigated with two-dimensional optical imaging spectroscopy in rat whisker barrel cortex. Eur J Neurosci 22:1655-1666.

Berwick J, Johnston D, Jones M, Martindale J, Martin C, Kennerley AJ, Redgrave P, Mayhew JE (2008) Fine detail of neurovascular coupling revealed by spatiotemporal analysis of the hemodynamic response to single whisker stimulation in rat barrel cortex. J Neurophysiol 99:787-798.

Boas DA, Jones SR, Devor A, Huppert TJ, Dale AM (2008) A vascular anatomical network model of the spatio-temporal response to brain activation. Neuroimage 40:1116-1129.

Chevalier G, Deniau JM (1990) Disinhibition as a basic process in the expression of striatal functions. Trends Neurosci 13:277-280.

Dahlhaus R, Kurths J, Maas P, Timor J (2008) Mathematical methods in signal processing and digital image analysis, $\mathrm{p} 42$. New York: Springer Publishing.

Derdikman D, Hildesheim R, Ahissar E, Arieli A, Grinvald A (2003) Imaging spatiotemporal dynamics of surround inhibition in the barrels somatosensory cortex. J Neurosci 23:3100-3105.

Devor A, Tian P, Nishimura N, Teng IC, Hillman EM, Narayanan SN, Ulbert I, Boas DA, Kleinfeld D, Dale AM (2007) Suppressed neuronal activity and concurrent arteriolar vasoconstriction may explain negative blood oxygenation level-dependent signal. J Neurosci 27:4452-4459.

Devor A, Hillman EM, Tian P, Waeber C, Teng IC, Ruvinskaya L, Shalinsky MH, Zhu H, Haslinger RH, Narayanan SN, Ulbert I, Dunn AK, Lo EH,
Rosen BR, Dale AM, Kleinfeld D, Boas DA (2008) Stimulus-induced changes in blood flow and 2-deoxyglucose uptake dissociate in ipsilateral somatosensory cortex. J Neurosci 28:14347-14357.

Di S, Baumgartner C, Barth DS (1990) Laminar analysis of extracellular field potentials in rat vibrissa/barrel cortex. J Neurophysiol 63:832-840.

Editorial (2009) Connecting the dots. Nat Neurosci 12:99.

Esclapez M, Campistron G, Trottier S (1987) Immunocytochemical localization and morphology of GABA-containing neurons in the prefrontal and frontoparietal cortex of the rat. Neurosci Lett 77:131-136.

Friston KJ, Frith CD, Liddle PF, Frackowiak RS (1991) Comparing functional (PET) images: the assessment of significant change. J Cereb Blood Flow Metab 11:690-699.

Golanov EV, Yamamoto S, Reis DJ (1994) Spontaneous waves of cerebral blood flow associated with a pattern of electrocortical activity. Am J Physiol 266:R204-R214.

Gonchar YA, Johnson PB, Weinberg RJ (1995) GABA-immunopositive neurons in rat neocortex with contralateral projections to S-I. Brain Res 697:27-34.

Harel N, Lee SP, Nagaoka T, Kim DS, Kim SG (2002) Origin of negative blood oxygenation level-dependent fMRI signals. J Cereb Blood Flow Metab 22:908-917.

Hewson-Stoate N, Jones M, Martindale J, Berwick J, Mayhew J (2005) Further nonlinearities in neurovascular coupling in rodent barrel cortex. Neuroimage 24:565-574.

Higley MJ, Contreras D (2007) Cellular mechanisms of suppressive interactions between somatosensory responses in vivo. J Neurophysiol 97:647-658.

Kastrup A, Baudewig J, Schnaudigel S, Huonker R, Becker L, Sohns JM, Dechent P, Klingner C, Witte OW (2008) Behavioral correlates of negative BOLD signal changes in the primary somatosensory cortex. Neuroimage 41:1364-1371.

Kennerley AJ, Berwick J, Martindale J, Johnston D, Papadakis N, Mayhew JE (2005) Concurrent fMRI and optical measures for the investigation of the hemodynamic response function. Magn Reson Med 54:354-365.

Logothetis NK, Pfeuffer J (2004) On the nature of the BOLD fMRI contrast mechanism. Magn Reson Imaging 22:1517-1531.

Logothetis NK, Wandell BA (2004) Interpreting the BOLD signal. Annu Rev Physiol 66:735-769.

Martin C, Jones M, Martindale J, Mayhew J (2006) Haemodynamic and neural responses to hypercapnia in the awake rat. Eur J Neurosci 24:2601-2610.

Martindale J, Mayhew J, Berwick J, Jones M, Martin C, Johnston D, Redgrave P, Zheng Y (2003) The hemodynamic impulse response to a single neural event. J Cereb Blood Flow Metab 23:546-555.

McDonald CT, Burkhalter A (1993) Organization of long-range inhibitory connections with rat visual cortex. J Neurosci 13:768-781.

Mitzdorf U (1985) Current source-density method and application in cat cerebral cortex: investigation of evoked potentials and EEG phenomena. Physiol Rev 65:37-100.

Muthukumaraswamy SD, Edden RA, Jones DK, Swettenham JB, Singh KD (2009) Resting GABA concentration predicts peak gamma frequency and fMRI amplitude in response to visual stimulation in humans. Proc Natl Acad Sci U S A 106:8356-8361.

Nakai M, Maeda M (1999) Scopolamine-sensitive and resistant components of increase in cerebral cortical blood flow elicited by periaqueductal gray matter of rats. Neurosci Lett 270:173-176.

Nicholson C, Freeman JA (1975) Theory of current source-density analysis and determination of conductivity tensor for anuran cerebellum. J Neurophysiol 38:356-368.

Northoff G, Walter M, Schulte RF, Beck J, Dydak U, Henning A, Boeker H, Grimm S, Boesiger P (2007) GABA concentrations in the human anterior cingulate cortex predict negative BOLD responses in fMRI. Nat Neurosci 10:1515-1517.

Pasley BN, Inglis BA, Freeman RD (2007) Analysis of oxygen metabolism implies a neural origin for the negative BOLD response in human visual cortex. Neuroimage 36:269-276.

Rauch A, Rainer G, Logothetis NK (2008) The effect of a serotonin-induced dissociation between spiking and perisynaptic activity on BOLD functional MRI. Proc Natl Acad Sci U S A 105:6759-6764.

Schridde U, Khubchandani M, Motelow JE, Sanganahalli BG, Hyder F, Blumenfeld H (2008) Negative BOLD with large increases in neuronal activity. Cereb Cortex 18:1814-1827. 
Shmuel A, Yacoub E, Pfeuffer J, Van de Moortele PF, Adriany G, Hu X, Ugurbil K (2002) Sustained negative BOLD, blood flow and oxygen consumption response and its coupling to the positive response in the human brain. Neuron 36:1195-1210.

Shmuel A, Augath M, Oeltermann A, Logothetis NK (2006) Negative functional MRI response correlates with decreases in neuronal activity in monkey visual area V1. Nat Neurosci 9:569-577.

Smith AT, Williams AL, Singh KD (2004) Negative BOLD in the visual cortex: evidence against blood stealing. Hum Brain Mapp 21:213-220.

Stefanovic B, Warnking JM, Pike GB (2004) Hemodynamic and metabolic responses to neuronal inhibition. Neuroimage 22:771-778.

Sun QQ, Huguenard JR, Prince DA (2006) Barrel cortex microcircuits: thalamocortical feedforward inhibition in spiny stellate cells is mediated by a small number of fast-spiking interneurons. J Neurosci 26: $1219-1230$.

Thomsen K, Offenhauser N, Lauritzen M (2004) Principal neuron spiking: neither necessary nor sufficient for cerebral blood flow in rat cerebellum. J Physiol 560:181-189.

Tomioka R, Okamoto K, Furuta T, Fujiyama F, Iwasato T, Yanagawa Y, Obata K, Kaneko T, Tamamaki N (2005) Demonstration of long-range GABAergic connections distributed throughout the mouse neocortex. Eur J Neurosci 21:1587-1600.

Uludağ K, Dubowitz DJ, Yoder EJ, Restom K, Liu TT, Buxton RB (2004) Coupling of cerebral blood flow and oxygen consumption during physiological activation and deactivation measured with fMRI. Neuroimage 23:148-155.

Wong-Riley MT, Welt C (1980) Histochemical changes in cytochrome oxidase of cortical barrels after vibrissal removal in neonatal and adult mice. Proc Natl Acad Sci U S A 77:2333-2337.

Zhang ZW, Deschênes M (1997) Intracortical axonal projections of lamina VI cells of the primary somatosensory cortex in the rat: a single-cell labeling study. J Neurosci 17:6365-6379. 